Deviation from equilibrium conditions in molecular dynamic simulations of homogeneous nucleation

\title{
Halonen, Roope
}

2018-04-28

Halonen , R , Zapadinsky , E \& Vehkamaki , H 2018 , ' Deviation from equilibrium conditions in molecular dynamic simulations of homogeneous nucleation ' , Journal of Chemical Physics, vol. 148 , no. 16,164508 . https://doi.org/10.1063/1.5023304

http://hdl.handle.net/10138/274998

https://doi.org/10.1063/1.5023304

acceptedVersion

Downloaded from Helda, University of Helsinki institutional repository.

This is an electronic reprint of the original article.

This reprint may differ from the original in pagination and typographic detail.

Please cite the original version. 


\title{
Deviation from equilibrium conditions in molecular dynamic simulations of homogeneous nucleation
}

\author{
Roope Halonen, ${ }^{1, \text { a) }}$ Evgeni Zapadinsky, ${ }^{1}$ and Hanna Vehkamäki ${ }^{1}$ \\ Institute for Atmospheric and Earth System Research / Physics, Faculty of Science, \\ University of Helsinki, Finland
}

(Dated: 7 August 2018)

We present a comparison between Monte Carlo (MC) results for homogeneous vapourliquid nucleation of Lennard-Jones clusters and previously published values from molecular dynamics (MD) simulations. Both the MC and MD methods sample real cluster configuration distributions. In the MD simulations the extent of the temperature fluctuation is usually controlled with an artificial thermostat rather than with more realistic carrier gas. In this study primarily velocity scaling thermostat is considered, but also Nosé-Hoover, Berendsen and stochastic Langevin thermostat methods are covered. The nucleation rates based on a kinetic scheme and the canonical MC calculation serve as a point of reference since they by definition describe an equilibrated system. The studied temperature range is from $T=0.3$ to $0.65 \epsilon / k$. The kinetic scheme reproduces well the isothermal nucleation rates obtained by Wedekind et al. [J. Chem. Phys. 127, 064501 (2007)] using MD simulations with carrier gas. The nucleation rates obtained by artificially thermostatted MD simulations are consistently lower than the reference nucleation rates based on MC calculations. The discrepancy increases up to several orders of magnitude when the density of the nucleating vapour decreases. At low temperatures the difference to the MC-based reference nucleation rates in some cases exceeds the maximal nonisothermal effect predicted by classical theory of Feder et al. [Adv. Phys. 15, 111 (1966)].

a)Electronic mail: roope.halonen@helsinki.fi 


\section{INTRODUCTION}

Nucleation is the limiting stage of first order phase transitions involving formation of stable embryos of the new phase. Predicting and controlling nucleation rate can help both developing new technologies and understanding natural phenomena: production of catalyst powders in chemical industry, formation of metal clusters in semiconductor design as well as ice crystal and aerosol particle formation in the atmosphere are examples of processes that involve nucleation.

Quantitative description of nucleation is hindered by the inconvenient scale of the process. The number of molecules in the embryo of the new phase is neither small enough to be described by a microphysical approach nor big enough to be pertinent for macrophysical bulk theories. Another difficulty is posed by strong heat exchange when nucleation occurs imposing need to control temperature in experiments and molecular simulations. Deviations from constant temperature have to be taken into account also in the theoretical development.

Although the first kinetic theory of vapour to liquid nucleation based on works Farkas, Szilard, Becker and Döring, and Zeldovich ${ }^{1-3}$ emerged about 80 years ago there are still many uncertainties and discrepancies within and between theories, simulations and experiments. In the present paper we use the term standard kinetic approach to refer to the most popular kinetic theory of nucleation usually called Becker-Döring or Szilard-Farkas approach, although contribution from other authors is significant as discussed in literature ${ }^{4,5}$.

Molecular dynamics (MD) simulations are often used for studying the nucleation process ${ }^{6-26}$. Nucleation can be studied directly and indirectly with $\mathrm{MD}^{9,18-20}$, in the present work only direct nucleation simulations are used. There are different methods for calculating the nucleation rate from MD simulations, and they have been shown ${ }^{17,21}$ to give similar results. MD approaches are free from assumptions that are invoked in nucleation theories concerning the treatment of equilibrium and idealistic bulk liquid nature of the clusters. In that sense MD simulations of the nucleation process can be used as a "numerical experiment" allowing testing of theoretical assumptions.

Many applications of gas-liquid nucleation theory concern situations where temperature of nucleating system is kept constant by collisions with the carrier gas. In MD simulations temperature is most often controlled by thermostats. Thermostats can however remove or add heat in unphysical manner, and the best procedure for the temperature control in MD 
simulations is to thermostat only the carrier $\operatorname{gas}^{7,15}$.

Monte Carlo (MC) simulation is another useful tool for studying the nucleation process. Unlike MD simulations which can capture the non-equilibrium features of the forming clusters, canonical MC results by definition refer to fully equilibrated clusters. However, the nucleation rate calculations from MC simulations invoke the assumptions made in the standard kinetic approach.

Nucleation rate should be identical in MD and MC simulations as long as the approximations made in the standard kinetic scheme are correct and MD simulations are really successful in modelling the isothermal conditions. The most frequent MD studies deal with Lennard-Jones argon. This has motivated us to study the nucleation of Lennard-Jones argon using standard kinetic approach with the work of cluster formation calculated by semi-grand canonical MC method ${ }^{27,28}$. We present here the comparison of the nucleation rates derived from MC simulations to the ones obtained by Tanaka et al., Diemand et al., Wedekind et al., Zhukhovitskii and Napari et al. using MD simulations ${ }^{15,20,22,23,26}$. We study the performance of different thermostatting methods in MD simulations of gas-liquid phase transition, and compare the difference between MD and MC -based results to the predictions of the classical nonisothermal nucleation theory. A short description of the standard kinetic approach is presented in the following section.

\section{STANDARD KINETIC APPROACH}

The kinetic scheme of nucleation is based on the picture that the vapour consists of clusters of different sizes. The clusters are characterised by number of monomers $n$, which can be molecules or atoms. They can experience evaporation and condensation. The heat released or absorbed by the clusters due to these processes is taken away by collision with carrier gas so that nucleation takes place at isothermal conditions. Provided the clusters detach and attach only monomers we can write death-birth equations for the number concentrations of $n$-clusters $N_{n}$ as

$$
\frac{\partial N_{n}}{\partial t}=\beta_{n-1} N_{n-1}+\alpha_{n+1} N_{n+1}-\beta_{n} N_{n}-\alpha_{n} N_{n}
$$

where $\beta_{n}$ is the monomer condensation rate on an $n$-cluster, $\alpha_{n}$ is the evaporation rate of a monomer from the cluster. The kinetic rates can be derived assuming a thermodynamic equilibrium: in a steady state (no net fluxes) the concentrations satisfy the detailed balance 
equation

$$
\beta_{n} N_{n}^{\mathrm{eq}}=\alpha_{n+1} N_{n+1}^{\mathrm{eq}}
$$

where superscript eq denotes that the number of $n$-clusters correspond to the equilibrium, Applying the detailed balance from cluster size 1 to $n$ one can write

$$
N_{n}^{\mathrm{eq}}=N_{1}^{\mathrm{eq}} \exp \left(-\frac{\Delta W_{n}}{k T}\right),
$$

where $k$ is the Boltzmann constant, $T$ is the temperature, and $\Delta W_{n}$ is the work of $n$-cluster formation.

Nucleation, that is the formation of large clusters, is possible when $\Delta W_{n}$ has maximum as in Figure 1. If $\Delta W_{n}$ increases monotonously with size, the number of $n$-clusters tends to zero with growing $n$. The size at which the work of formation has maximum is called the critical size $n^{*}$. Nucleation rate can be expressed in terms of condensation rates as ${ }^{17,29}$

$$
J=\left[\sum_{n=1}^{\bar{n}} \frac{1}{\beta_{n} N_{n}^{\mathrm{eq}}}\right]^{-1} .
$$

Alternatively, using the detailed balance equation (Eq. 2) we can rearrange the equation for the nucleation rate calculation to involve the evaporation rates

$$
J=\left[\sum_{n=1}^{\bar{n}} \frac{1}{\alpha_{n+1} N_{n+1}^{\mathrm{eq}}}\right]^{-1} .
$$

The practical choice of the size limit $\bar{n}$ is defined by desired accuracy. If $\bar{n}=n^{*}$, we use critical size as the limit, we underestimate the nucleation rate by a factor of approximately 2. It is usually enough to use $\bar{n}=a n^{*}$ with factor $a$ ranging from 1.15 to 1.30 depending on $n^{*}$. The cluster size distribution in the nucleating vapour can be related to the equilibrium cluster concentration and the nucleation rate as ${ }^{17,29}$

$$
N_{n+1}=N_{n+1}^{\mathrm{eq}}\left(1-J \sum_{n=1}^{n} \frac{1}{\alpha_{n+1} N_{n+1}^{\mathrm{eq}}}\right) .
$$

Cluster distributions in the nucleating vapour and in equilibrium are shown in Figure 1.

\section{THEORETICAL DETAILS}

Eqs. (3) and (4) of the previous section create a basis for the nucleation rate calculation, but we still need to know equilibrium cluster distribution $N_{n}^{\text {eq }}$ and condensation $\left(\beta_{n}\right)$ or evaporation rates $\left(\alpha_{n}\right)$. Besides that we need to consider the case when concentration of carrier gas is too low to effectively thermalize the nucleating clusters. 

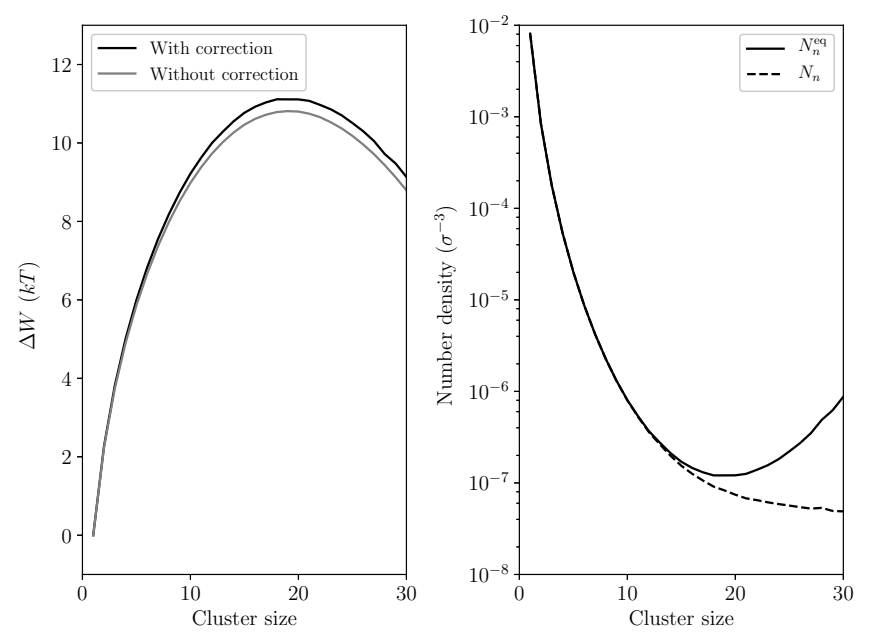

FIG. 1. Left: Work of cluster formation calculated with Monte Carlo at $T=0.6 \epsilon / k\left(N_{\text {tot }}=\right.$ $0.0108 \sigma^{-3}$ ) with the vapour-cluster interaction correction $\Delta \omega_{n}$ (black line, see supplementary material) and without (grey line). Right: Cluster concentrations in the nucleating vapour (dashed line) and the equilibrium cluster concentrations (solid line) for corrected $\Delta W_{n}$.

\section{A. Equilibrium cluster distribution}

There are a wide variety of methods for obtaining cluster work of formation and the equilibrium cluster distribution. The historically first approach is the liquid drop model ${ }^{30}$ where the clusters are presented as tiny spheres of bulk liquid. Combined with the standard kinetic approach it constitutes the so-called classical nucleation theory. The liquid drop model has been modified several times using different phenomenological methods including density functional theory ${ }^{31-33}$.

Another family of methods is based on the cluster free energy calculation by means of statistical mechanics. The cluster free energy can be calculated by separating a cluster motion into translational, rotational and vibrational modes. In this case to calculate vibrational and rotational partition functions the best option is to use Quantum Chemistry. Another approach to calculate a cluster free energy is to perform integration over the classical phase space. In the latter case Monte Carlo methods are used. In addition to the molecular interaction model, these methods require the cluster criterion, which can be introduced through parameters in the simulations ${ }^{34-36}$ or defined self-consistently ${ }^{37-39}$.

The main goal of the present study is the comparison of the nucleation rates based on standard kinetic approach to results of MD numerical experiments. Therefore we choose the 
cluster criteria and methods of calculating potential energy of the clusters identical to the MD simulations.

There are several MC methods which calculate the work of cluster formation $\Delta W_{n}{ }^{27,28,35,36,40-48}$ from a given interacting scheme; these methods produce essentially identical results ${ }^{48,49}$. In this work $\Delta W_{i}$ is calculated by semi-grand canonical Metropolis Monte Carlo method $27,28,46$. This method calculates canonical ensemble average of the grand canonical growth and decay probabilities for a single cluster size at the time. The average growth and decay probabilities, $\bar{G}_{i}$ and $\bar{D}_{i}$, obtained from the simulation allow us to calculate the work of formation

$$
\Delta W_{n}=-k T \sum_{i=2}^{n} \ln \frac{\bar{G}_{i-1}}{\bar{D}_{i}} .
$$

The MC simulation results calculated at one monomer density $N_{1}^{(1)}$ can be easily scaled to obtain the work of formation at some other monomer density $N_{1}^{(2)} 46$ :

$$
\frac{\bar{G}_{i-1}\left(N_{1}^{(2)}\right)}{\bar{D}_{i}\left(N_{1}^{(2)}\right)}=\frac{N_{1}^{(2)}}{N_{1}^{(1)}} \frac{\bar{G}_{i-1}\left(N_{1}^{(1)}\right)}{\bar{D}_{i}\left(N_{1}^{(1)}\right)} .
$$

Thus, for each temperature the simulation has to be performed only once.

The MC method neglects the vapour-cluster interaction. However, some of the MD simulations have been performed at such conditions that the justification of this approximation needs to be assessed. We have used recipe of Oh and Zeng ${ }^{50}$ to study this effect and the correction $\Delta \omega_{n}$ to Eq. (7) is given in the supplementary material. The used correction term is significant only at high temperatures 0.6 and $0.65 \epsilon / k(\approx 72$ and $78 \mathrm{~K}$, respectively) but even then the correction to the work of the cluster formation is minor (see Figure 1). For lower temperatures $\Delta \omega_{n}$ is negligible.

\section{B. Evaporation rate}

Eq. (4) is much more often used for the calculation of the nucleation rate than Eq. (5). The attachment rates are usually taken as the cluster-monomer collision frequencies from the kinetic gas theory. However, evaporation rates obtained from MD simulation ${ }^{51}$ provide more reliable data than the kinetic gas theory-based condensation rates relying on the liquid density rather than the densities of the real clusters. Still, the obtained evaporation rates are only 2-6 times higher than the rates estimated by Eq. (2) using the kinetic theory ${ }^{51,52}$. The evaporations rate $\alpha_{n}(E)$ of the $n$-clusters can be obtained in MD 
simulations in the microcanonical ensemble ( $n V E$, where $V$ is volume and $E$ is energy $)^{52-54}$. The averaged detachment rate calculated over the Maxwell-Boltzmann distribution $\varphi_{n}(E)$ give the isothermal evaporation rate of the cluster

$$
\alpha_{n}=\int_{-\infty}^{\infty} \varphi_{n}(E) \alpha_{n}(E) d E .
$$

Inserting these evaporation rates $\alpha_{n}$ into Eq. (5) gives us the nucleation rate.

\section{Classical theory for nonisothermal nucleation rate}

As latent heat is released in the condensation or the evaporative cooling, the clusters' energy variation can differ from the Maxwell-Boltzmann distribution at ambient temperature if the thermalization is not efficient enough. In the theory of Feder et al. ${ }^{4}$ the nonisothermality is characterized by modelling growth in size and energy space. The energy gained by a cluster by addition of a monomer is given as

$$
q=h-\frac{k T}{2}-\gamma \frac{\partial A_{n}}{\partial n},
$$

where $h$ is the macroscopic latent heat, $\gamma$ is the surface tension and $A_{n}$ is the surface area of the $n$-cluster. The thermodynamic parameterisations for the latent heat, surface tension and density of LJ liquid can be found e.g. from the Appendix A of Ref. 23. During the time interval between subsequent size changes the latent heat can be removed by collisions with vapour or carrier gas molecules. The mean square energy fluctuation of the colliding ideal monatomic vapour and gas molecules can be estimated as ${ }^{15}$

$$
b^{2}=2 k^{2} T^{2}\left(1+\frac{N_{\mathrm{c}}}{N_{1}} \sqrt{\frac{m}{m_{\mathrm{c}}}}\right),
$$

where $N_{\mathrm{c}}$ is the densities of the carrier gas, $m$ and $m_{\mathrm{c}}$ are the masses of the molecules of condensible vapour and the carrier gas, respectively.

According to the nonisothermal nucleation theory the nucleation rate is given as the isothermal nucleation rate multiplied with a correction factor:

$$
J_{\text {noniso }}=\frac{b^{2}}{b^{2}+q^{2}} J_{\text {iso }} .
$$

The nonisothermal nucleation rate approaches the isothermal rate when the ratio $b / q$ is high, which represent a case where the amount of carrier gas is relatively high and the carrier gas particles are comparably light. 
Other versions of the nonisothermal nucleation theories ${ }^{55-58}$ give quantitatively similar results to the Feder et al. formula.

\section{COMPUTATIONAL DETAILS}

\section{A. Molecular Dynamics}

There are many MD studies related to homogeneous vapour to liquid nucleation simulations. Among these we have chosen for comparison only those for which we could unambiguously extract data necessary for analogous MC simulations and thus perform a solid comparison to our MC simulation results. Each of these recent MD studies model the same nucleation phenomenon for the same system, but the treatment of thermal equilibration of the system and the methods to obtain the nucleation rate are different. The simulated particles interact via the Lennard-Jones (LJ) potential

$$
u(r)=4 \epsilon\left[\left(\frac{\sigma}{r}\right)^{12}-\left(\frac{\sigma}{r}\right)^{6}\right],
$$

where $\epsilon$ and $\sigma$ are the argon Lennard-Jones parameters, $r$ is the distance between atoms. The potential is truncated after varying distances and the tail of the potential is often shifted to zero after the truncation distance.

In the numerical simulations the cluster definition of Stillinger ${ }^{34}$ is used. The cluster definition requires that each atom/molecule in a cluster has at least one neighbour within a certain connectivity distance $R_{\mathrm{st}}$ to belong to the same cluster.

The MD study at high supersaturation and for relatively small systems $\left(10^{4}\right.$ to $2 \times 10^{5}$ particles in a volume region from $2 \times 10^{6}$ to $8.8 \times 10^{8} \sigma^{3}$ ) by Tanaka et al. ${ }^{22}$ and the MD study at low supersaturation with large amount of particles $\left(10^{9}\right.$ and $8 \times 10^{9}$ in a volume region from $1.6 \times 10^{10}$ to $1.3 \times 10^{13} \sigma^{3}$ ) by Diemand et al. ${ }^{23}$ at temperature range of $T=0.2$ to $1.0 \mathrm{\epsilon} / k$ followed similar procedures. In these large-scale simulations the canonical ensemble was approximated by using the velocity scaling (VS) thermostat scheme: the system is instantaneously adjusted to the desired temperature by rescaling the velocities of particles after every time step $\Delta t$. Here the LJ potential was truncated and shifted to zero at $5 \sigma$ and the Stillinger connectivity distances are defined for every temperature separately (see Table II in Ref. 23). The nucleation rate was calculated by the method of Yasuoka and Matsumoto ${ }^{7}$. 
Zhukhovitskii ${ }^{26}$ has studied nucleation in the system with number of particles from $2.68 \times$ $10^{5}$ to $6.25 \times 10^{5}$ in volumes $1 \times 10^{9}$ and $8 \times 10^{9} \sigma^{3}$ at temperature of $0.65 \epsilon / k$. His study used different thermostatting methods for monomers and clusters. The monomers are subject to the modified Berendsen thermostat ${ }^{6}$, which scales the velocities less abruptly than the instantaneous VS thermostat, and it also accelerates/decelerates individual monomers if they are below/above the average energy corresponding to the desired temperature. Stochastic Langevin thermostat was used for the clusters to model the collisions with an imaginary carrier gas. Furthermore, two additional simulation cases were carried out without the cluster thermostatting. The supersaturation was kept nearly constant by introducing new monomers into the system and removing clusters from the system after they have reached some threshold size. The nucleation rate was calculated from the removal rate of the clusters beyond the threshold size. Napari et al. ${ }^{20}$ also run simulations at $0.65 \epsilon / k$ (2300 particles in a volume of $\left.(168.191 \sigma)^{3}\right)$ so that the whole system is under Berendsen thermostat and the nucleation rate was obtained by the mean first-passage time (MFPT) method ${ }^{16}$ instead $^{2}$ of direct observation method ${ }^{17}$. MFPT and direct observation method result in similar nucleation rates in gas-liquid nucleation of Lennard-Jones atoms ${ }^{21}$. In both Zhukhovitskii and Napari et al. studies the simulation were carried out for particles with the LJ potential truncated and shifted at $2.5 \sigma$ with Stillinger connectivity distance of $1.5 \sigma$. (Note that Zhukhovitskii uses time unit $\sigma \sqrt{m / 24 \epsilon}$ instead of commonly used $\sigma \sqrt{m / \epsilon}$.)

In the last comparable MD nucleation study of Lennard-Jones molecules Wedekind et al. ${ }^{15}$ used thermostatted carrier gas (LJ helium) at $50 \mathrm{~K}(\approx 0.42 \epsilon / k)$ instead of intensive thermostat to mimick a realistic nucleation event. Their system size was much smaller than in the MD studies mentioned above, only 343 condensable atoms in volumes $(16 \mathrm{~nm})^{3}$ and $(18 \mathrm{~nm})^{3}$, and they detected the nucleation rate using the MFPT method. Beside its realistic nature, a further advantage of using carrier gas is the possibility to link the results to the nonisothermal nucleation theory ${ }^{4}$ as the nonisothermal nucleation rate depends on the ratio between the amount of carrier gas and condensable atoms (Eq. (12)). In addition to simulations with carrier gas they also used VS and Nosé-Hoover thermostats; virtually the two different thermostat schemes yield identical results at chosen conditions so for brevity only VS is considered here. They demonstrated that a longer time step and lower velocity scaling frequency lead to more effective thermalization of the system until a point is reached beyond which the thermostatting is too infrequent or the trajectories of atoms 
are unphysical. Wedekind et al. use only truncated potential without shifting at $5 \sigma$ with parameters $\sigma=3.405 \AA$ and $\epsilon / k=120 \mathrm{~K}$. The Stillinger connectivity distance is now $1.8 \sigma$.

\section{B. Monte Carlo}

To adequately compare the results calculated with MC and MD methods, we use matching Lennard-Jones potentials and cluster criteria for each comparison MC run.

In the actual comparison between $\mathrm{MD}$ and $\mathrm{MC}$ results the monomer depletion has to be taken into account as about 1 to $50 \%$ of the molecules are clustered in the MD simulations when a quasi-steady state is reached in Refs. 22 and 23. Accordingly, in nucleation rate calculations with MC data the total number of nucleating atoms, expressed as

$$
N_{\text {tot }}=\sum_{n=1}^{\bar{n}} n N_{n},
$$

is set to match the number density of corresponding MD simulation. The nucleating vapour concentrations are then calculated with Eq. (6).

There are two reasons to choose $N_{\text {tot }}$ to be identical in comparing the results of MD and MC simulations instead of more frequently used saturation ratio defined as $S=P_{1} / P_{1 \text { s }}$, where $P_{1}$ and $P_{1 \mathrm{~s}}$ are the partial monomer pressures at actual and saturated vapour conditions, respectively. First, the number of monomers is often not reported in the MD papers,

only the total number of nucleating molecules (atoms) is given . So, knowing the volume of the simulation box the total number concentration of nucleating atoms $N_{\text {tot }}$ can be calculated, but number of free monomers $N_{1}$ is unknown due to depletion. Second, the thermostats used in MD simulations do not necessarily provide fully isothermal conditions for nucleation, the temperature dependence of the saturation ratio leads to additional uncertainty in the comparison.

\section{RESULTS AND DISCUSSION}

The nucleation rates are calculated from the MC data using Eq. (5) to correspond to selected set of temperatures and number densities used in MD simulations listed earlier. The calculation of the evaporation rates limits the number of systems that are available for the comparison: at very low energies the long lifetimes of clusters and also large critical 
size $n^{*}$ increase considerably the computational effort. In addition, at very low densities the determination of the nucleation rate from MD simulation can be challenging (in simulations

of Diemand et al. ${ }^{23}$ at some densities no stable clusters has emerged and the nucleation rates of such cases has been derived from the Poisson distribution) and thus the comparison is limited to higher densities.

The size limit $\bar{n}$ was selected individually for different temperatures bearing in mind that the computational effort should be reasonable and further increasing $\bar{n}$ should not affect the nucleation rate substantially. Figure 1 shows that $N_{n}^{\text {eq }}$ increases exponentially after the critical size, and thus Eq. (5) rapidly converges.

All the MC simulations are carried out initially at monomer concentration $N_{1}$ of $0.0012 \sigma^{-3}$ and then scaled with Eq. (8) to appropriate concentration. The results are reported in Table I with the reference MD data.

\section{A. Depletion of free monomers}

In the MC-based calculations the free monomer concentration $N_{1}$ is determined iteratively so that the total number of atoms $N_{\text {tot }}$ (see Eq.s (6) and (14)) equals the total number of nucleating atoms used in the MD simulations with the accuracy of $0.1 \%$. Note that in case of comparison with Zhukhovitskii ${ }^{26}$ this is not necessary because the number of monomers is kept constant during the MD simulations and the reported $N_{\text {tot }}$ in Table I are calculated using Eq. (6). In the case of Tanaka et al. ${ }^{22}$ the level of depletion in the MD simulation can be easily estimated from the reported initial saturation ratio $S_{0}$ and the average saturation ratio in the nucleation stage $S$. The depletion as a function of number density for the MC scheme calculated using the standard kinetic scheme and the MD simulations of Tanaka et al. is presented in Figure 2. The results show that the level of depletion is quite well modelled by the standard kinetic scheme especially at high temperatures and at low densities. The estimated depletion at highest density at $T=$ $0.3 \epsilon / k$ in the MD simulation has counterintuitive value: the depletion is decreased while the density and thus the nucleation rate increase. In general determining the average pressure at the nucleation stage can be difficult in MD simulations because the cluster distribution of the system can evolve quite rapidly and the partial pressure of monomers can fluctuate considerably. It should be noted that most of the "depleted" monomers are attached to the 
TABLE I. Summary of the results of the MC and MD simulations sorted according to the MD studies of Diemand et al. ${ }^{23}(\mathrm{D}(2013))$, Tanaka et al. ${ }^{22}(\mathrm{~T}(2011))$, Wedekind et al. ${ }^{15}(\mathrm{~W}(2007))$, Zhukhovitskii $^{26}$ (Z(2016) with both Berendsen and Langevin thermostats B $+\mathrm{L}$ and with only Berendsen B) and Napari et al. ${ }^{20}(\mathrm{~N}(2009))$. Given are temperature $T$, total numbed density $N_{\text {tot }}$, nucleation rate by using $\mathrm{MC}$ data $J_{\mathrm{MC}}$ and obtained with $\mathrm{MD}$ simulation $J_{\mathrm{MD}}$, critical cluster sizes $n_{\mathrm{MC}}$ and $n_{\mathrm{MD}}$ obtained with MC and MD, respectively, depletion of monomers $D$ and used boundary condition in the standard kinetic scheme $\bar{n}$.

\begin{tabular}{|c|c|c|c|c|c|c|c|c|c|}
\hline & $T(\epsilon / k)$ & $N_{\text {tot }}\left(\sigma^{-3}\right)$ & & $J_{\mathrm{MC}}\left(\sigma^{-3} \tau^{-1}\right)$ & $J_{\mathrm{MD}}\left(\sigma^{-3} \tau^{-1}\right)$ & $n_{\mathrm{MC}}^{*}$ & $n_{\mathrm{MD}}^{*}$ & $\mathrm{D}(\%)$ & $\bar{n}$ \\
\hline \multirow[t]{3}{*}{$\mathrm{D}(2013)$} & 0.30 & $9.00 \times 10^{-5}$ & & $2.24 \times 10^{-16}$ & $5.30 \times 10^{-20}$ & 10 & 15 & 1.2 & 17 \\
\hline & 0.30 & $1.20 \times 10^{-4}$ & & $4.37 \times 10^{-15}$ & $1.56 \times 10^{-17}$ & 9 & 14 & 1.7 & \\
\hline & 0.30 & $1.40 \times 10^{-4}$ & & $1.95 \times 10^{-14}$ & $1.32 \times 10^{-16}$ & 9 & 13 & 1.9 & \\
\hline \multirow[t]{4}{*}{$\mathrm{T}(2011)$} & 0.30 & $2.28 \times 10^{-4}$ & & $1.42 \times 10^{-12}$ & $3.00 \times 10^{-14}$ & 7 & 6 & 3.3 & \\
\hline & 0.30 & $3.70 \times 10^{-4}$ & & $4.76 \times 10^{-11}$ & $1.30 \times 10^{-12}$ & 6 & 5 & 7.4 & \\
\hline & 0.30 & $6.40 \times 10^{-4}$ & & $6.09 \times 10^{-10}$ & $3.50 \times 10^{-11}$ & 6 & 5 & 23.6 & \\
\hline & 0.30 & $1.08 \times 10^{-3}$ & & $2.34 \times 10^{-9}$ & $5.50 \times 10^{-10}$ & 5 & 4 & 44.6 & \\
\hline \multirow[t]{3}{*}{$\mathrm{D}(2013)$} & 0.40 & $6.00 \times 10^{-4}$ & & $5.95 \times 10^{-16}$ & $9.54 \times 10^{-18}$ & 16 & 15 & 3.8 & 30 \\
\hline & 0.40 & $7.00 \times 10^{-4}$ & & $6.54 \times 10^{-15}$ & $8.99 \times 10^{-17}$ & 15 & 14 & 4.4 & \\
\hline & 0.40 & $1.00 \times 10^{-3}$ & & $8.67 \times 10^{-13}$ & $1.49 \times 10^{-14}$ & 12 & 12 & 6.3 & \\
\hline \multirow[t]{5}{*}{$\mathrm{T}(2011)$} & 0.40 & $1.35 \times 10^{-3}$ & & $2.75 \times 10^{-11}$ & $4.00 \times 10^{-12}$ & 11 & 8 & 8.6 & \\
\hline & 0.40 & $1.35 \times 10^{-3}$ & & $2.75 \times 10^{-11}$ & $8.00 \times 10^{-12}$ & 11 & 8 & 8.6 & \\
\hline & 0.40 & $1.71 \times 10^{-3}$ & & $2.72 \times 10^{-10}$ & $1.50 \times 10^{-11}$ & 9 & 7 & 11.6 & \\
\hline & 0.40 & $2.78 \times 10^{-3}$ & & $6.18 \times 10^{-9}$ & $6.50 \times 10^{-10}$ & 8 & 6 & 25.8 & \\
\hline & 0.40 & $3.70 \times 10^{-3}$ & & $1.69 \times 10^{-8}$ & $6.00 \times 10^{-9}$ & 7 & 5 & 37.8 & \\
\hline \multirow[t]{2}{*}{$\mathrm{W}(2007)$} & 0.42 & $2.31 \times 10^{-3}$ & & $8.36 \times 10^{-10}$ & $4.48 \times 10^{-11}$ & 10 & 14.1 & 18.1 & 30 \\
\hline & 0.42 & $3.29 \times 10^{-3}$ & & $7.76 \times 10^{-9}$ & $9.21 \times 10^{-10}$ & 9 & 13.2 & 29.1 & \\
\hline \multirow[t]{3}{*}{$\mathrm{D}(2013)$} & 0.50 & $2.60 \times 10^{-3}$ & & $5.26 \times 10^{-14}$ & $5.26 \times 10^{-16}$ & 21 & 23 & 9.6 & 30 \\
\hline & 0.50 & $3.20 \times 10^{-3}$ & & $2.41 \times 10^{-12}$ & $6.15 \times 10^{-14}$ & 18 & 20 & 11.8 & \\
\hline & 0.50 & $4.00 \times 10^{-3}$ & & $7.63 \times 10^{-11}$ & $2.74 \times 10^{-12}$ & 16 & 18 & 14.6 & \\
\hline \multirow[t]{4}{*}{$\mathrm{T}(2011)$} & 0.50 & $5.00 \times 10^{-3}$ & & $1.31 \times 10^{-9}$ & $7.00 \times 10^{-11}$ & 13 & 9 & 18.4 & \\
\hline & 0.50 & $5.00 \times 10^{-3}$ & & $1.31 \times 10^{-9}$ & $1.00 \times 10^{-10}$ & 13 & 9 & 18.4 & \\
\hline & 0.50 & $6.40 \times 10^{-3}$ & & $1.39 \times 10^{-8}$ & $2.00 \times 10^{-9}$ & 12 & 8 & 24.4 & \\
\hline & 0.50 & $7.23 \times 10^{-3}$ & 12 & $3.27 \times 10^{-8}$ & $6.00 \times 10^{-9}$ & 11 & 8 & 28.5 & \\
\hline $\mathrm{D}(2013)$ & 0.60 & $6.50 \times 10^{-3}$ & & $1.47 \times 10^{-13}$ & $2.58 \times 10^{-15}$ & 32 & 38 & 15.7 & 38 \\
\hline
\end{tabular}


smallest clusters far below the critical size.

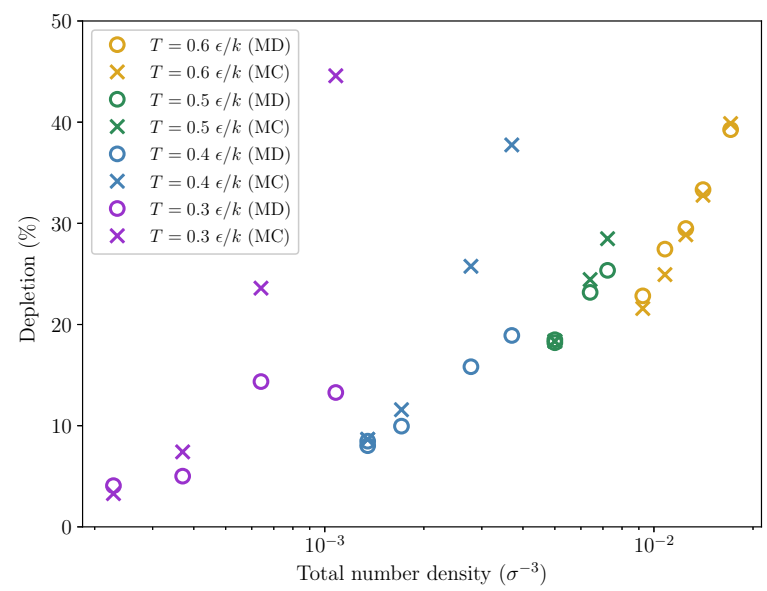

FIG. 2. Depletion of monomers as a function of total number density for MD (circles) $)^{22}$ and MC simulations (crosses) at different temperatures.

\section{B. Nonisothermality as a function of carrier gas concentration}

In the MD simulations of Wedekind et al. ${ }^{15}$ nucleation occurs in nonisothermal conditions with a known amount of carrier gas. The ratio of Wedekind et al. nucleation rate to MCbased results for an identical system can be compared to the the prediction of the classical theory of nonisothermal nucleation, Eq. (12). Figure 3 shows that the ratio of the MD and $\mathrm{MC}$-based results is very close to the prediction of the classical theory. In the more dense system the rates are about two times higher than predicted by the theory. Moreover, the effect of nonisothermality predicted by the theory is quite moderate, about one to two orders of magnitude.

\section{Comparison of $\mathrm{MC}$ results to $\mathrm{MD}$ simulations that use velocity scaling}

The comparison between the isothermal nucleation rates obtained with the MC data and the MD simulated nucleation rates of Tanaka et $a .^{22}$ and Diemand et $a l .{ }^{23}$ using velocity scaling thermostat are shown in Figure 4 (Tanaka: coloured circles and Diemand: coloured squares). The MD nucleation rates $J_{\mathrm{MD}}$ are uniformly lower than the ones based on the MC simulations, and the ratio $J_{\mathrm{MD}} / J_{\mathrm{MC}}$ increases toward unity when the concentration $N_{\text {tot }}$ and the temperature increase. 


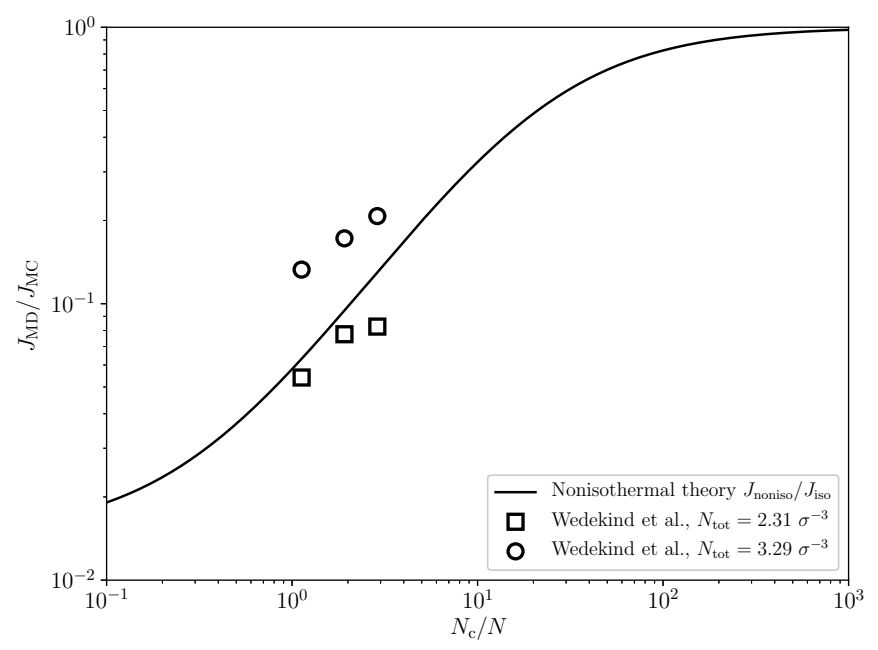

FIG. 3. Ratio of the nucleation rates of Wedekind et al. ${ }^{15}$ and the MC-based calculations as a function of the ratio of carrier gas and condensable monomer densities at $50 \mathrm{~K}(\approx 0.42 \epsilon / k)$. The solid line shows the ratio of the nonisothermal and isothermal nucleation rates based on classical theory, Eq. (12).

The effect of the time step in MD simulations using velocity scaling is demonstrated by Wedekind et al. ${ }^{15}$ and it is shown in Figure 4: tenfold increase in $\Delta t$ results in over two times higher nucleation rate. Tanaka et al. ${ }^{22}$ simulated a system with identical densities using both $10^{4}$ and $10^{5}$ atoms (at $T=0.4 \epsilon / k, N_{\text {tot }}=1.35 \times 10^{-3}$ and $T=0.5 \epsilon / k, N_{\text {tot }}=5 \times 10^{-3}$ ) and the nucleation rates are about twice higher with less atoms. The difference can be due to the lack of gathered statistics: the nucleation rate is determined from the population of stable clusters.

In the absence of carrier gas $\left(N_{\mathrm{c}}=0\right)$ the nonisothermal factor given by Eq. (12) is naturally smallest and it is illustrated with the coloured bars in Figure 4 for different temperatures. The level of this effect is lower at high temperatures since the energy fluctuation term $b$ is proportional to temperature. The energy released in an addition of a monomer to an $n$-cluster ( $q$ in Eq. (12)) is slightly increasing with $n$ and this decreases the nonisothermal factor only marginally for larger critical cluster sizes. Thus, for clarity, we show the nonisothermal factor only for the lowest density used in the MD simulations at each temperature in Figure 4.

It has been shown that the most probable cluster "temperatures" in MD simulations are at least qualitatively compatible with the nonisothermal nucleation theory ${ }^{15,59}$ : at low 
temperature the cluster temperatures differ significantly from the bath temperature but at high temperature the difference is small. The theory states that this is due to the energy fluctuation of the impinging molecules $b$ which is proportional to $T$.

Since the level of the velocity scaling depends on the excess heat, the cooling of the system is more effective when there is a substantial amount of "hot" clusters. In the presence of few clusters i.e. at very low monomer density the ratio $J_{\mathrm{MD}} / J_{\mathrm{MC}}$ is even less than the the theoretical value of the maximal nonisothermal effect calculated without any thermalizing agent. At high monomer density, due to a high concentration of overheated clusters and considerable monomer depletion, the overall temperature after one time step is relatively high which results in more substantial removal of the latent heat from the clusters by the thermostat. This is inline with the result that for higher densities the difference between MD and MC nucleation rates is smaller. Another possible explanation of the smaller discrepancy between MD and MC results at higher vapour densities is that the nucleating vapour itself can serve as natural thermostat for the clusters due to non-sticking collisions.

In experiment of Sinha et al. ${ }^{60}\left(J=10^{17 \pm 1} \mathrm{~cm}^{-3} \mathrm{~s}^{-1}\right)$ the vapour pressures varies from 0.47 to $8 \mathrm{kPa}$ at temperature range from 34 to $53 \mathrm{~K}$ (number density is about $4 \times 10^{-5}$ $\left.4 \times 10^{-4} \sigma^{-3}\right)$ and in experiments of Fladerer et al. and Iland et al. ${ }^{61,62}\left(J=10^{7 \pm 2} \mathrm{~cm}^{-3} \mathrm{~s}^{-1}\right)$

the pressures are between 0.3 to $10 \mathrm{kPa}$ at $42-59 \mathrm{~K}$ (about $2 \times 10^{-5}-4 \times 10^{-4} \sigma^{-3}$ ). They are substantially lower than in the MD simulations. Also MD studies of nucleation in the presence of carrier gas are performed only at carrier gas concentrations comparable to that of the nucleating Lennard-Jones vapour. Thus, fully isothermal conditions are not reached in the simulations. Deeper understanding of the nucleation process require additional MD, MC and theoretical studies.

\section{Comparison of $\mathrm{MC}$ results to $\mathrm{MD}$ simulations with separate thermostats for monomers and clusters}

Comparison of MC-based results to MD simulations calculated using the separate thermostatting scheme for monomers and clusters is presented in Figure 5. As in the case of the VS thermostatted results, the ratio $J_{\mathrm{MD}} / J_{\mathrm{MC}}$ increases as the density increases also in this case. The difference grows even steeper with decreasing number density than in Figure 4. When using stochastic Langevin thermostat for the clusters only, nonisothermal effects 


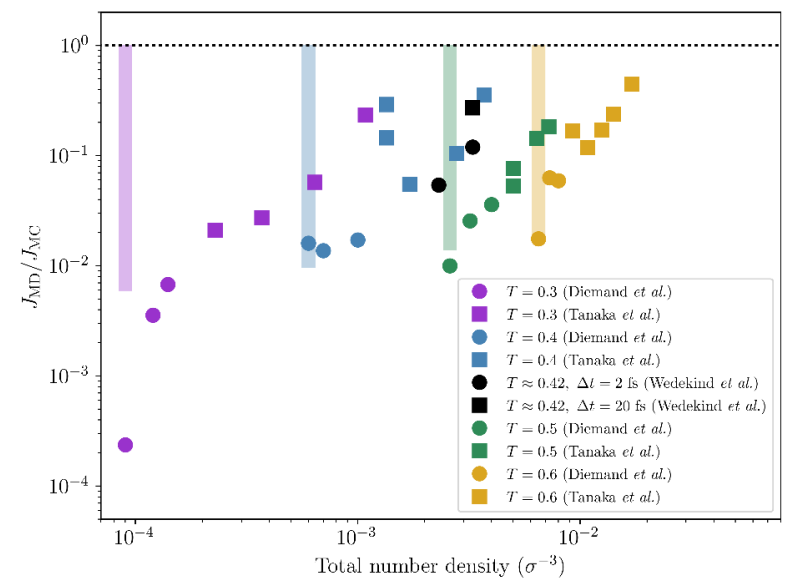

FIG. 4. Ratio $J_{\mathrm{MD}} / J_{\mathrm{MC}}$ as a function of total monomer density where $J_{\mathrm{MD}}$ are obtained by Tanaka et al. ${ }^{22}$ (coloured squares), Diemand et al..$^{23}$ (coloured circles) and Wedekind et al. ${ }^{15}$ (black markers) using velocity scaling thermostat. The coloured bars represent the maximal $J_{\text {noniso }} / J_{\text {iso }}$ ratio according to the nonisothermal nucleation theory.

can be present because the thermostat subjects the clusters to imaginary collisions at some finite rate.

If only the monomers are thermostatted, their velocities correspond to some specific temperature and the set-up corresponds to that of classical nonisothermal nucleation theory at the limit of no carrier gas. Indeed, without thermalization of clusters the MD nucleation rates are considerably lower.

The nucleation rate calculated using Berendsen thermostat for the whole system ${ }^{20}$ matches very well the rates calculated with separate cluster thermostatting when the depletion of monomers is taken into account.

\section{CONCLUSIONS}

In the present study we have compared published results of MD simulations to the results of the standard kinetic approach where the work of the cluster formation has been calculated using a Monte Carlo approach. We observe good agreement only with simulations of Wedekind et al. ${ }^{15}$ where carrier gas has been used as a thermostat, when the correction factor of the classical nonisothermal nucleation theory ${ }^{4}$ is used with the standard kinetic 


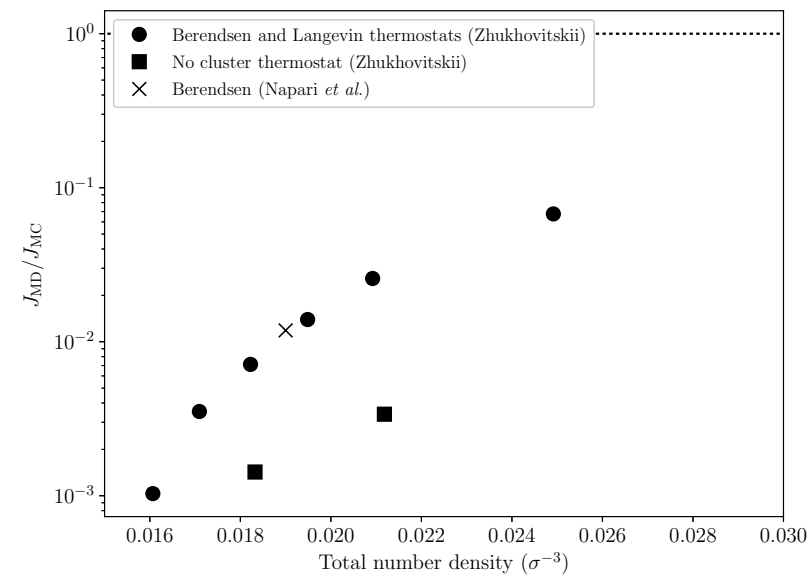

FIG. 5. Ratio between the nucleation rate from the MD of Zhukhovitskii ${ }^{26}$ and the nucleation rate obtained by MC data (circles and squares) and Napari et al. ${ }^{20}$ (a cross). Thermostatting of the system is treated either with separate Berendsen thermostat for the monomers and Langevin thermostat for the clusters (circles), only monomers are thermostatted with Berendsen thermostat (squares) or the whole system is thermostatted with Berendsen thermostat (a cross).

scheme. The standard kinetic scheme predicts the nucleation rate within a factor of two, and to our knowledge this is currently the most accurate test of the scheme since the work of cluster formation is calculated with $\mathrm{MC}$ method where the interaction between atoms is identical to the MD simulations.

MD simulations performed with more artificial thermostats (velocity scaling, Berendsen, Langevin and Nosé-Hoover) yield nucleation rates even further away from the MC-based results. The discrepancy increases when the nucleating vapour decreases. In some cases the difference can exceed the maximal factor predicted by the nonisothermal theory. These thermostats do not completely thermalize the system, since most of them remove or add heat equally from/to all atoms, although only the atoms bound to clusters heat up or cool down in the cluster formation or decay processes ${ }^{7,15}$. However, the sources of disagreement can also be assumptions in the standard kinetic approach such as that work of the cluster formation can be described by a thermodynamical formula and that the clusters in the metastable equilibrium and nucleating vapour have equivalent structure. These assumptions have already been questioned in literature ${ }^{20,29,59,63}$. 


\section{SUPPLEMENTARY MATERIAL}

See supplementary material for the derivation of the vapor-cluster interaction.

\section{ACKNOWLEDGMENTS}

This work was supported by the European Research Council (Grant 692891-DAMOCLES) and University of Helsinki, Faculty of Science ATMATH project. The authors wish to acknowledge CSC - IT Center for Science, Finland, for computational resources.

\section{REFERENCES}

${ }^{1}$ L. Farkas, Z. Physik. Chem. 125, 236 (1927).

${ }^{2}$ R. Becker and W. Döring, Ann. Phys. 24, 719 (1935).

${ }^{3}$ J. Zeldovich, Zh. Eksp. Theor. Fiz. 12, 525 (1942).

${ }^{4}$ J. Feder, K. Russell, J. Lothe, and G. Pound, Adv. Phys. 15, 111 (1966).

${ }^{5}$ D. Kashchiev, Nucleation: Basic theory with applications (Butterworth-Heinemann, Oxford, 2000).

${ }^{6}$ D. Zhukhovitskii, J. Chem . Phys 103, 9401 (1995).

${ }^{7}$ K. Yasuoka and M. Matsumoto, J. Chem. Phys. 109, 8451 (1998).

${ }^{8}$ K. Yasuoka and M. Matsumoto, J. Chem. Phys. 109, 8463 (1998).

${ }^{9}$ K. Laasonen, S. Wonczak, R. Strey, and A. Laaksonen, J. Chem. Phys. 113, 9741 (2000).

${ }^{10}$ S. Toxvaerd, J. Chem. Phys. 115, 8913 (2001).

${ }^{11}$ S. Toxvaerd, J. Chem. Phys. 119, 10764 (2003).

${ }^{12}$ K. Tanaka, K. Kawamura, H. Tanaka, and K. Nakazawa, J. Chem. Phys. 122, 184514 (2005).

${ }^{13}$ N. Lümmen and T. Kraska, J. Aerosol Sci. 36, 1409 (2005).

${ }^{14}$ T. Kraska, J. Chem. Phys. 124, 054507 (2006).

${ }^{15}$ J. Wedekind, D. Reguera, and R. Strey, J. Chem. Phys. 127, 064501 (2007).

${ }^{16}$ J. Wedekind, R. Strey, and D. Reguera, J. Chem. Phys. 126, 134103 (2007).

${ }^{17}$ J. Julin, I. Napari, and H. Vehkamäki, J. Chem. Phys. 126, 224517 (2007).

${ }^{18}$ M. Horsch, J. Vrabec, and H. Hasse, Phys. Rev. E 78, 011603 (2008).

${ }^{19}$ J. Julin, I. Napari, J. Merikanto, and H. Vehkamäki, J. Chem. Phys. 129, 234506 (2008). 
${ }^{20}$ I. Napari, J. Julin, and H. Vehkamäki, J. Chem. Phys. 131, 244511 (2009).

${ }^{21}$ G. Chkonia, J. Wölk, R. Strey, J. Wedekind, and D. Reguera, J. Chem. Phys. 130, 064505 (2009).

${ }^{22}$ K. K. Tanaka, H. Tanaka, T. Yamamoto, and K. Kawamura, J. Chem. Phys. 134, 204313 (2011).

${ }^{23}$ J. Diemand, R. Angélil, K. K. Tanaka, and H. Tanaka, J. Chem. Phys. 139, 074309 (2013).

${ }^{24}$ S. Toxvaerd, J. Chem. Phys. 143, 154705 (2015).

${ }^{25}$ S. Toxvaerd, J. Chem. Phys. 144, 164502 (2016).

${ }^{26}$ D. Zhukhovitskii, J. Chem. Phys. 144, 184701 (2016).

${ }^{27}$ H. Vehkamäki and I. J. Ford, J. Chem. Phys. 112, 4193 (2000).

${ }^{28}$ J. Merikanto, H. Vehkamaki, and E. Zapadinsky, J. Chem. Phys. 121, 914 (2004).

${ }^{29}$ E. Zapadinsky, J. Chem. Phys. 135, 194504 (2011).

${ }^{30}$ M. Volmer and A. Weber, Z. Phys. Chem. 119, 277 (1925).

${ }^{31}$ D. W. Oxtoby and R. Evans, J. Chem. Phys. 89, 7521 (1988).

${ }^{32}$ A. Laaksonen and D. W. Oxtoby, J. Chem. Phys. 102, 5803 (1995).

${ }^{33}$ I. Napari, A. Laaksonen, and V. Talanquer, J. Chem. Phys. 110, 5906 (1999).

${ }^{34}$ F. H. Stillinger Jr, J. Chem. Phys. 38, 1486 (1963).

${ }^{35}$ J. K. Lee, J. A. Barker, and F. F. Abraham, J. Chem. Phys. 58, 3166 (1973).

${ }^{36}$ P. R. ten Wolde and D. Frenkel, J. Chem. Phys. 109, 9901 (1998).

${ }^{37}$ H. Reiss, A. Tabazadeh, and J. Talbot, J. Chem. Phys. 92, 1266 (1990).

${ }^{38}$ G. K. Schenter, S. M. Kathmann, and B. C. Garrett, Phys. Rev. Lett. 82, 3484 (1999).

${ }^{39}$ G. K. Schenter, S. Kathmann, and B. Garrett, J. Chem. Phys. 110, 7951 (1999).

${ }^{40}$ G. M. Torrie and J. P. Valleau, Chem. Phys. Lett. 28, 578 (1974).

${ }^{41}$ C. H. Bennett, J. Comput. Phys. 22, 245 (1976).

${ }^{42}$ N. G. Garcia and J. M. Soler Torroja, Phys. Rev. Lett. 47, 186 (1981).

${ }^{43}$ B. N. Hale and R. Ward, J. Stat. Phys. 28, 487 (1982).

${ }^{44}$ A. P. Lyubartsev, A. Laaksonen, and P. N. Vorontsov-Velyaminov, Mol. Phys. 82, 455 (1994).

${ }^{45}$ E. Zapadinsky and M. Kulmala, J. Chem. Phys. 102, 6858 (1995).

${ }^{46}$ A. Lauri, J. Merikanto, E. Zapadinsky, and H. Vehkamäki, Atm. Res. 82, 489 (2006).

${ }^{47}$ B. Chen, J. I. Siepmann, K. J. Oh, and M. L. Klein, J. Chem. Phys. 115, 10903 (2001). 
${ }^{48}$ B. Chen, H. Kim, S. J. Keasler, and R. B. Nellas, J. Phys. Chem. B 112, 4067 (2008).

${ }^{49}$ J. Merikanto, E. Zapadinsky, A. Lauri, and H. Vehkamäki, Phys. Rev. Lett. 98, 145702 (2007).

${ }^{50}$ K. J. Oh and C. Zeng, X, J. Chem. Phys. 112, 294 (2000).

${ }^{51}$ R. Halonen, E. Zapadinsky, and H. Vehkamäki, (unpublished manuscript).

52 J. C. Barrett, J. Chem. Phys. 126, 074312 (2007).

${ }^{53}$ S. A. Harris and I. J. Ford, J. Chem. Phys. 118, 9216 (2003).

${ }^{54}$ I. Napari and H. Vehkamäki, J. Chem. Phys. 124, 024303 (2006).

${ }^{55}$ A. P. Grinin and F. M. Kuni, Theor. Math. Phys. 80, 968 (1989).

${ }^{56}$ B. E. Wyslouzil and J. H. Seinfeld, J. Chem. Phys. 97, 2661 (1992).

${ }^{57}$ J. Barrett, J. Phys. A: Math. Gen. 27, 5053 (1994).

${ }^{58}$ J. C. Barrett, J. Chem. Phys. 128, 164519 (2008).

${ }^{59}$ R. Angélil, J. Diemand, K. K. Tanaka, and H. Tanaka, J. Chem. Phys. 140, 074303 (2014).

${ }^{60}$ S. Sinha, A. Bhabhe, H. Laksmono, J. Wölk, R. Strey, and B. Wyslouzil, J. Chem. Phys. 132, 064304 (2010).

${ }^{61}$ A. Fladerer and R. Strey, J. Chem. Phys. 124, 164710 (2006).

${ }^{62}$ K. Iland, J. Wölk, R. Strey, and D. Kashchiev, J. Chem. Phys. 127, 154506 (2007).

${ }^{63}$ L. Bartell, J. Chem. Phys. 131, 174505 (2009). 